\title{
The Improvement of Weighted Least Square State Estimation Accuracy Using Optimal PMU Placement
}

\author{
KHALED AL-MAITAH, BATOOL AL-KHRIESAR, ABDULLAH AL-ODIENAT \\ Department of Electrical Engineering, Faculty of Engineering \\ Mutah University, Karak, Postal Code (61710), Jordan \\ JORDAN
}

\begin{abstract}
This paper proposed a new technique to improve the state estimation performance by optimal placement of phasor measurement units (OPP), the proposed technique is based on Simulating Annealing (SA) algorithm for OPP by comparing between the SA solution sets and choosing the optimal location of PMUs to enhance the state estimation performance. The proposed technique has been tested through IEEE 24 bus test system using power system analysis toolbox in MATLAB program. In this paper the root mean squared deviation (RMSD) has been used to determine the state estimation performance, the simulation result demonstrates that the proposed method proved its effectiveness to be one of the best methods used to improve the state estimation performance.
\end{abstract}

Key-Words: Phasor measurement unit, State estimation, Estimation Accuracy, Optimal placement of PMU, Simulating Annealing.

Received: March 8, 2019. Revised: December 23, 2019. Accepted: January 11, 2020. Published: January $18,2020$.

\section{Introduction}

The classical state estimation of a power system is based upon measurements composed from a supervisory control and data acquisition (SCADA) system. Such system cannot capture measurements of fast and dynamic phenomena. Another stumbling block of SCADA systems, it does not include the angle of bus voltages or branch currents. Otherwise, real-time voltage value and current phasors can be measured by phasor measurement units (PMUs). These are digital devices with very high sampling rate and precision, synchronized by receiving a common timing signal from a global positioning satellite (GPS) clock [1].

The PMUs receive its synchronized signals from the GPS Satellite, and are now being manufactured commercially. PMUs importance has presented because it can provide synchronized measurements of real-time currents and phasor of voltage to the state estimator [2].

Many of PMUs have required improving the controllability, monitoring, and the protection of the power system, but it is not credible to place a PMU at each bus of the power system, because the PMUs cost and communication services is so expensive. A desired number of PMUs are needed to be built up at certain locations which make the system fully observable. This is an optimization problem known as the optimal location of PMUs.

References [3-7] give an inclusive review of the problem in the literature. There are related works in the domain of PMU optimization present in many paper. Moreover, further information on objective functions, constraints, and optimization methods tried by researchers. PMUs have many significant applications, and one of them is to apply PMUs in electrical power systems for improving the state estimation performance and accuracy [8]. Power system state estimation forms the backbone of the Energy Management System (EMS), which plays important role in monitoring and controlling power systems for reliable operations. A traditional state estimator estimates voltage magnitudes and phase angles at the buses by using obtainable measurements which including magnitudes of bus voltages, branches current, power injections, and power flows [9].

The estimator determines the better estimate of the current electrical power system states which may include the voltage phasors for each bus, tap positions of the transformer and the status of circuit breaker, using the flow of telemetry that has been obtained from the system's sensors, current system model and information from other data sources. In the modern control centers, state estimator is the major input for many system supervision applications

With installation of PMUs in power systems, the state estimator has two more measurements of current phasors and voltage, and it much more accurate than the classic measurements.

Many researchers have presented several ways to improve state estimation performance, in paper [10] authors apply the incremental placement algorithm to improve the estimation accuracy. In that paper the authors determined the estimation performance by using the covariance matrix of state vector from the inverse of the gain matrix in weighted least square (WLS) technique. Papers [11] discuss PMU placement 
algorithm and take advantage of PMU technology to improve the bad data processing capability of state estimation. Paper [12] proposes the enhancement of the state-estimation precision, authors used the performance indicator of mean average percentage error (MAPE) to determine the state estimation precision. Paper [13] discuss an optimization strategy of selecting PMU locations in the power system to improve the estimation accuracy as by covering the critical regions, authors suggest that by covering the critical zones during PMU placement will surely ensure the robustness of the estimation performance of the power system.

This paper proposed the optimal location of PMU by Annealing Method as well as to enhancement the state estimation performance. Root-mean-square deviation (RMSD) has been used to evaluate the state estimation precision. Flow chart has been built to illustrate the Suggested method to choose the optimal location set of PMU which reinforcement state estimation accuracy, while maintaining the system to be observable. IEEE 24-bus system is used to present the proposed method, Simulation results in this paper done by Power System Analysis Toolbox in MATLAB program.

Section 2 in this paper presents a brief discussion about phasor measurement unit structure and its optimal placement techniques, while section 3 presents a mathematical model of the weighted least square (WLS) state estimation.

Section 4 presents the proposed method to choose optimal location set of PMUs to enhance the state estimation performance, section 5 gives a case study to test the proposed method in IEEE 24 bus system and finally section 6 covers the results and discussion.

\section{Phasor Measurement Unit}

PMUs are now used in power systems for many prospect applications. PMU importance appears from its ability to supply synchronized measurements of real-time phasor of voltage and currents to the state estimator [14].

In this section the PMU structure and optimal placement of PMU have been briefly discussion.

\subsection{Phasor Measurement Unit Structure}

The PMU located at any bus can measure the phasor voltage of that bus (magnitude and angle) and as well as needed currents (magnitude and angle) of branches which connected with that bus.

The applications of the phase measuring units include; measuring voltage magnitude and phasors, frequency, and current. These measurement may used by state estimation to; instability prediction, improved control, and adaptive relaying.

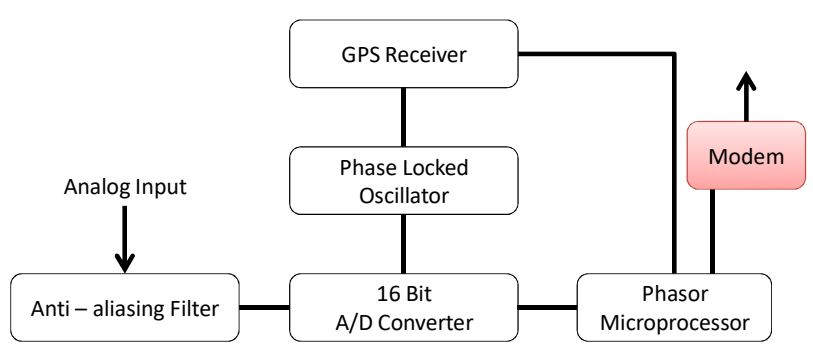

Fig. 1. Phasor measurement unit PMU structure.

State estimator is useful to monitor the state of the power system and it's widely used in modern electric power system utilities control systems. Several measurements, such as real and reactive powers and voltage or current magnitudes, received from different station are fed into the state estimator. Using a repeated nonlinear estimation procedure, the state estimator determines the power system state. Practically, by using PMUs, the state estimation can play a significant tool in stability and security of power system operations.

Consequently the PMU structure shown in fig. 1, the analog inputs are currents and voltages obtained from the secondary windings of the current and voltage transformers. The current and voltage signals are transformed to voltages with appropriate shunts or instrument transformers (current and voltage transformer) so that it are matched with the requirements of the analog to digital converters (ADC).

The sampling rate selection for the sampling operation dictates the frequency response of the antialiasing filters. Practically, these are analog type filters with a cut-off frequency less than half the sampling frequency in order to satisfy the Nyquest criterion. The sampling clock is phase locked with the GPS clock pulse. Even higher sampling rates are surely likely in the future leading to more accurate phasor estimates since higher sampling rates leading to improved estimation accuracy.

\subsection{Optimal placement of PMUs}

Since it is not economical to place PMUs at each bus, we need a placement technique which will provide a complete observability of the system. Placement techniques will depend on the PMUs application, installation restriction and the system characteristics as it is not even necessary also to place at each and every bus.

Many topological and numerical methods are there to determine the optimal location of PMUs; these may include Minimum Spanning Tree (MST), Depth First Search (DeFS), Simulated Annealing (SA), Tabu Search (TS) and others. The Simulated Annealing (SA) has been used in this paper to get the optimal locations set of PMU and then choosing the better solution which enhance the state estimation performance. 


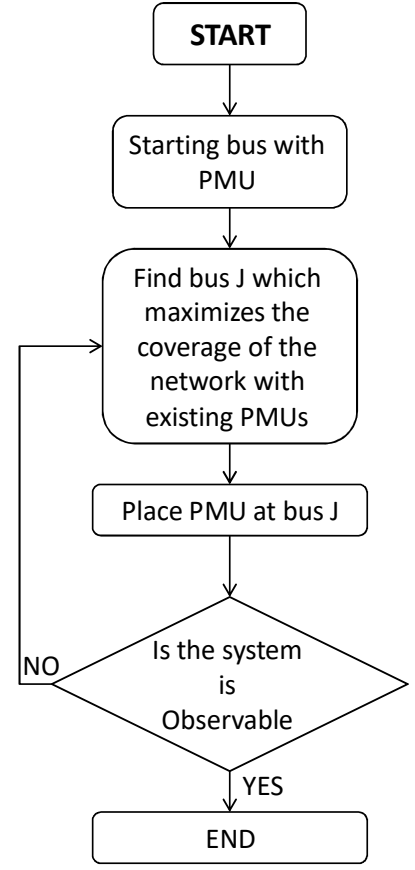

Fig. 2. Flow chart of simulated annealing technique

Simulated Annealing (SA) is a technique to finds a perfect solution to an optimization problem, by trying indiscriminate variations of the current solution. The technique can be divided into several main steps which are summarized in the flow chart shown in fig. 2.

\section{State Estimation}

The common method used to solve the static SE is weighted least square and weighted least absolute value methods; in this section the theoretical background of SE with weighted least square method is briefly covered.

\subsection{Weighted Least Square WLS State estimation}

State Estimation (SE) is mainly used to nominate excrescent data, to remove improper measurements and to make reliable state estimates. It allows the determination of the power flows in portion of the power system which are not directly measured.

Practically, the state variables of State estimation are the voltages and phase angles for each bus in the system. Once the estimates of the state variables are known the proper actions can be done by the system operator, if required (during emergency, normal insecure states), the system can be restored to its normal state.

Weighted Least Square (WLS) state estimator is a conventional state estimator to find the better state vector, A set of measurements specified by the vector $Z$, assumed to be expressed by the non-linear function of the state vectors and a vector of measurement errors, can be stated in compact matrix form as in equation (1).
$Z=\left[\begin{array}{c}z_{1} \\ z_{1} \\ \vdots \\ z_{m}\end{array}\right]=\left[\begin{array}{c}h_{1}\left(x_{1}, x_{2}, \ldots, x_{n}\right) \\ h_{2}\left(x_{1}, x_{2}, \ldots, x_{n}\right) \\ \vdots \\ h_{m}\left(x_{1}, x_{2}, \ldots, x_{n}\right)\end{array}\right]+\left[\begin{array}{c}e_{1} \\ e_{1} \\ \vdots \\ e_{m}\end{array}\right]=h(x)+e$

Where $\mathrm{Z}$ Measurement vector, $\mathrm{h}(\mathrm{x})$ is a non-linear function vector which relates measurements to states, $x$ State Vector, e the error vector between measured and estimated value. The measurements are presumed to be fully separate and independent of each other, and the measurement errors are independent also. The covariance matrix $\square \square \square$ is thus fully diagonal, i.e. $\square \square \square=\{\square 12, \square 12, \ldots, \square \square 2\}$, Where $\sigma \mathrm{i}$ is Variance of the ith measurement and $\mathrm{m}$ is a number of measurements.

Equation (2) show that the objective of the Weighted Least Square state estimator is to minimize the error e in the measurements i.e, is the sum of the squares of weighted residuals.

$J(x)=\sum_{i=1}^{m} \frac{\left(Z_{i}-h_{i}(x)\right)^{2}}{R_{i i}}$

$X_{k+1}=X_{k}+\left[[H]^{T}[R]^{-1}[H]\right]^{-1}[H]^{T}[R]^{-1}\left[\begin{array}{c}Z_{1}-h_{1}(x) \\ Z_{2}-h_{2}(x) \\ \vdots \\ \vdots \\ Z_{m}-h_{m}(x)\end{array}\right]$

A repeated solution can be used to find the state of the power system. A repeated solution scheme known as the Gauss Newton technique which neglecting the higher order terms of the Taylor series as in equation (3). The Jacobian matrix $[\mathrm{H}]$ will be acquired By considering a partial derivative of $\mathrm{h}(\mathrm{x})$ with respect to state vector $X .[R]$ is the error covariance matrix of measurements and $\mathrm{k}$ is an iterative number.

\subsection{Estimation Accuracy Assessment}

In the simulation offered in this paper, the root-meansquare deviation RMSD has been used to assess the deviation of estimates from the "exact" states acknowledging the knowledge of power system true states derived by power flow calculation. The RMSD for the voltage angles are in degree and the voltage magnitudes are in p.u.

In equation (4), the RMSD of an estimator voltage $\hat{v}$ with respect to an actual voltage $v$ is defined as the square root of the mean square error:

$R M S D=\sqrt{\operatorname{MSE}(\hat{v})}=\sqrt{E\left((\hat{v}-v)^{2}\right)}$

So the estimation accuracy by root-mean-square deviation (RMSD), defined by equation (5)

$R M S D=\sqrt{\frac{1}{n} \sum_{i=1}^{n}\left(v_{i}-\hat{v}_{i}\right)^{2}}$ 
Where $\mathrm{n}$ is a number of measurements, RMSD results are always non-negative, and a value of 0 (almost doesn't achieved in practice) would indicate a perfect fit to the data. In general, the state estimator which has lower RMSD is better than a higher one.

\section{The Proposed Method To Improve SE Performance}

The flow chart of Simulated Annealing SA technique produces the optimal location set of PMU, thus the proposed method use that set to choose the optimal location of PMU to improve state estimation accuracy and performance.

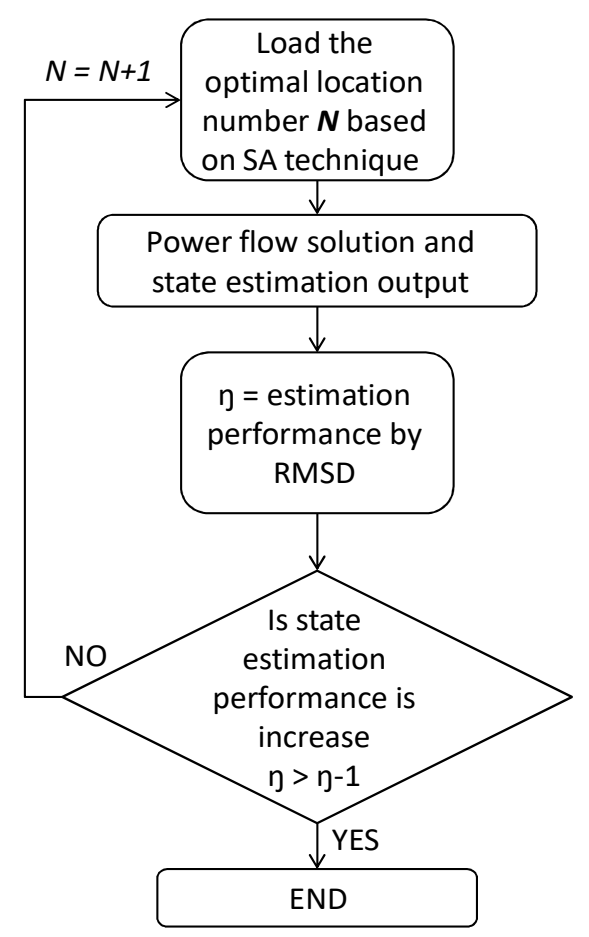

Fig. 3. Flow chart of proposed method

The proposed method is integrated with Simulated Annealing method, which means that the results obtained from Simulated Annealing method appear as the input of our method. The flow chart is shown in figure 3 where $\eta$ is a state estimation performance and $\mathrm{N}$ is number of Simulated Annealing solution set.

The flow chart clearly illustrate that the method start by loading the results of simulated annealing method, to ensure the least number of PMU which achieves the observability of the system, after all possible solution are loaded the power flow solution is then obtained by Newton-Raphson method and the state estimation output is also obtained to calculate the performance of state estimation by root mean square deviation RMSD. The proposed method determines the state estimation performance of all possible solution and compares all sets with respect to state estimation performance to obtain finally the optimal location of PMU which in turns significantly improves the state estimation performance. By this method all the system will be observable and the state estimation will be in the best performance with respect to other location.

The method can be applied to any electrical power system suffers from state estimation performance. Moreover it increases the state estimation robustness which will in turn enhance the stability of the power system through sending accurate information to the control center.

\section{Case Study}

To investigate the proposed method and check state estimation performance for different PMU sets, a IEEE 24 bus test system has been used to applied the proposed method and to determine the best location of phasor measurement unit which improve the state estimation performance. The 24-bus system, shown in figure.4, contains 24 buses, 10 generators, 3 power transformers and 17 loads.

Table 1. Power flow result for IEEE-24 bus system

\begin{tabular}{|c|c|c|c|c|c|}
\hline Bus & $\begin{array}{c}\mathbf{V} \\
{[\text { p.u] }}\end{array}$ & $\begin{array}{c}\text { phase } \\
{[\text { rad] }}\end{array}$ & Bus & $\begin{array}{c}\text { V } \\
{[\text { p.u] }}\end{array}$ & $\begin{array}{c}\text { phase } \\
{[\text { rad] }}\end{array}$ \\
\hline Bus01 & 1.035 & -0.127 & Bus13 & 1.020 & 0.000 \\
\hline Bus02 & 1.035 & -0.129 & Bus14 & 0.980 & 0.013 \\
\hline Bus03 & 0.983 & -0.087 & Bus15 & 1.014 & 0.227 \\
\hline Bus04 & 0.997 & -0.170 & Bus16 & 1.017 & 0.209 \\
\hline Bus05 & 1.017 & -0.175 & Bus17 & 1.038 & 0.287 \\
\hline Bus06 & 1.009 & -0.219 & Bus18 & 1.050 & 0.310 \\
\hline Bus07 & 1.025 & -0.131 & Bus19 & 1.023 & 0.176 \\
\hline Bus08 & 0.991 & -0.196 & Bus20 & 1.039 & 0.182 \\
\hline Bus09 & 0.999 & -0.131 & Bus21 & 1.050 & 0.325 \\
\hline Bus10 & 1.025 & -0.168 & Bus22 & 1.050 & 0.424 \\
\hline Bus11 & 0.991 & -0.047 & Bus23 & 1.050 & 0.197 \\
\hline Bus12 & 1.002 & -0.025 & Bus24 & 0.979 & 0.112 \\
\hline
\end{tabular}

\subsection{Newton Raphson load flow solution}

Firstly the test system has been solved in power flow solution by power system analysis toolbox to obtain the actual value of voltage magnitudes and phase angles for each bus in the system.

The Newton-Raphson load flow solution results which are represented as voltage magnitudes and phase angle for each bus shown in table 1, power flow results have been calculated at base power 100 MVA. 


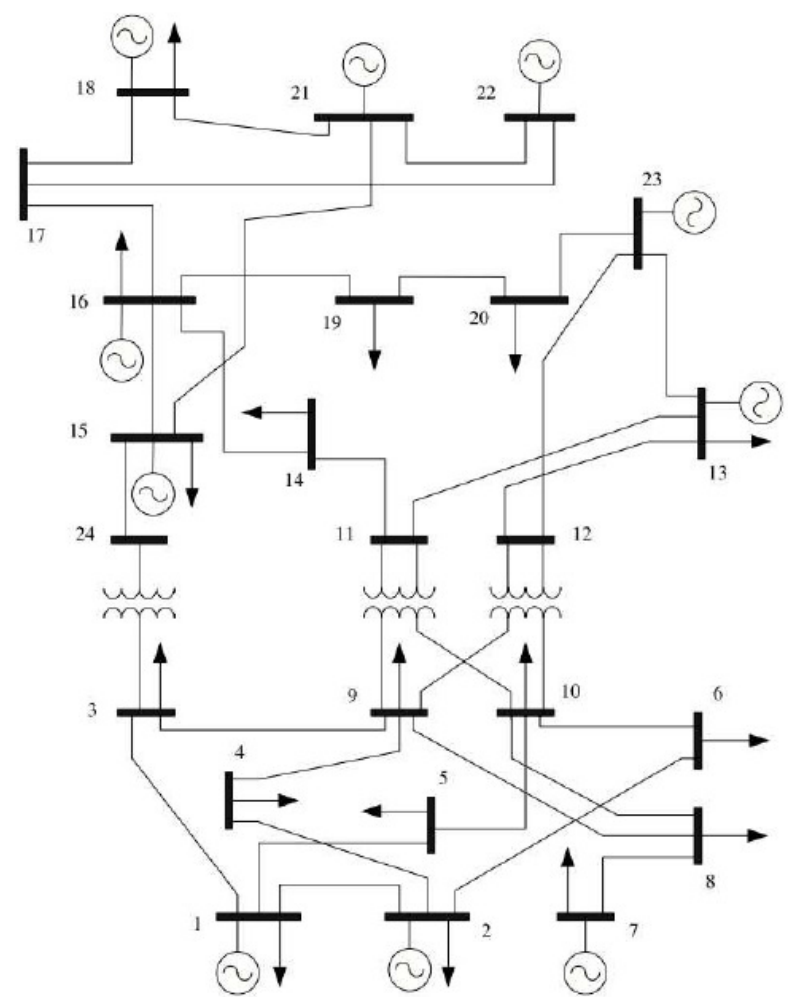

Fig. 4. Single line diagram of IEEE-24bus system

Table 2. PMU Optimal placement based on system observability

\begin{tabular}{|c|c|c|c|}
\hline Set & PMUs & Placement & $\begin{array}{c}\text { \#Non-observable } \\
\text { bus }\end{array}$ \\
\hline $\mathbf{1}$ & 6 & $1,2,8,9,15,17,20$ & 0 \\
\hline $\mathbf{2}$ & 6 & $2,8,10,15,20,22$ & 0 \\
\hline $\mathbf{3}$ & 6 & $2,8,10,15,20,22$ & 0 \\
\hline $\mathbf{4}$ & 6 & $2,8,10,15,17,20$ & 0 \\
\hline $\mathbf{5}$ & 7 & $1,2,8,9,15,17,20$ & 0 \\
\hline $\mathbf{6}$ & 7 & $3,4,8,10,20,21,22$ & 0 \\
\hline $\mathbf{7}$ & 7 & $2,8,10,15,16,22,23$ & 0 \\
\hline $\mathbf{8}$ & 7 & $2,8,10,11,15,20,22$ & 0 \\
\hline $\mathbf{9}$ & 7 & $1,6,8,9,15,17,20$ & 0 \\
\hline $\mathbf{1 0}$ & 7 & $2,8,10,15,16,18,20$ & 0 \\
\hline $\mathbf{1 1}$ & 7 & $1,4,8,10,15,20,22$ & 0 \\
\hline $\mathbf{1 2}$ & 7 & $1,8,9,10,15,17,20$ & 0 \\
\hline $\mathbf{1 3}$ & 7 & $1,2,8,13,15,17,20$ & 0 \\
\hline $\mathbf{1 4}$ & 7 & $3,4,8,10,17,20,21$ & 0 \\
\hline
\end{tabular}

\subsection{Optimal Placement of PMU}

The Simulated Annealing (SA) has been used to obtain the optimal placement set of PMU in IEEE 24 bus test system by power system analysis toolbox. The possible solution sets obtained for optimal PMU placement in IEEE 24 bus are shown in table 2. There are fourteen possible solutions. Actually, one solution gives an optimal improvement of state estimation performance, as shown in table 2 , all set has zero for non-observable bus, that means all solutions achieve the observability for the whole system.

\section{Results and Discussions}

The case study in this paper is IEEE 24 bus system of 24 buses as it is mentioned in previous section. Table 2 refers to PMU placement solution set, so the 24 bus system is fully observable with 6 or 7 PMUs only. With 7 PMUs the system will have more redundant measurement which means the state estimation will be more accurate than using 6 PMUs. The state estimation performance has been calculated for each solution set.

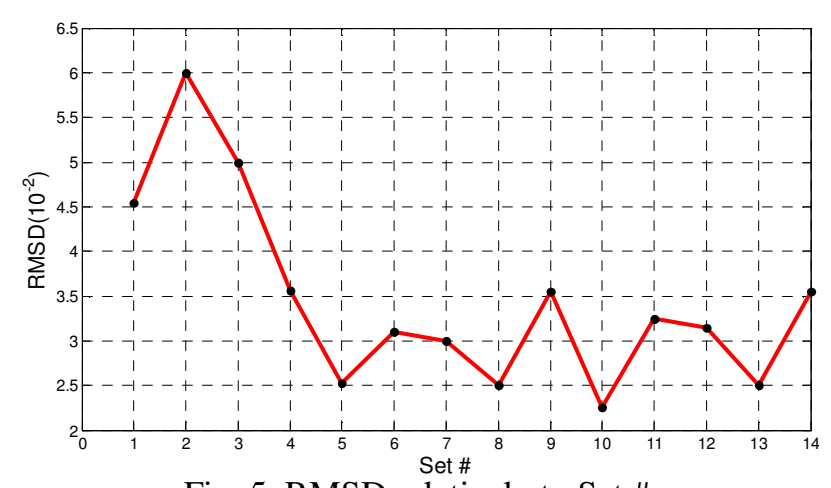

Fig. 5. RMSD relatively to Set \#

The state estimation performance has been calculated by RMSD to obtain the optimal location based on proposed method, which depends on state estimation performance.

From Fig.5, it can be observed that there is a major estimation improvement when PMU placed in set number ten, which means that with the PMUs installed at buses 2,8,10,15,16,18,20 the state estimation performance (determined by RMSD) will be about 0.022. This best PMUs locations from state estimation point of view. On the other hand, figure 5 demonstrates at set number 1,2,3,4 the state estimation performance lower than other sets that because at first four sets a six PMUs has been used, but at other sets seven PMUs has been used in system, that is mean the system with large number of PMUs has a good state estimation performance.

With seven PMUs installed in IEEE 24-bus system at the optimal location (set number \#10), the system is tested by applying a three phase to ground fault at bus number five, the clearing time of the fault has been set to $0.07 \mathrm{~s}$ at the simulation program. The results show a high accuracy of the state estimator as shown in Fig. 6. Fig. 7 clearly shows that the PMUs measurement of voltage responses at bus 10 is very close to the actual responses. The maximum difference of voltage response at bus 10 is about 0.05 p.u and the maximum difference of voltage response at bus 2 is about 0.1 p.u. Fig. 8 demonstrate theta response at bus 10 after a the 
three phase to ground fault is applied at bus 5. It is clear that the PMU measurements at this disturbance is again very close the actual response which was drawn intermittently.

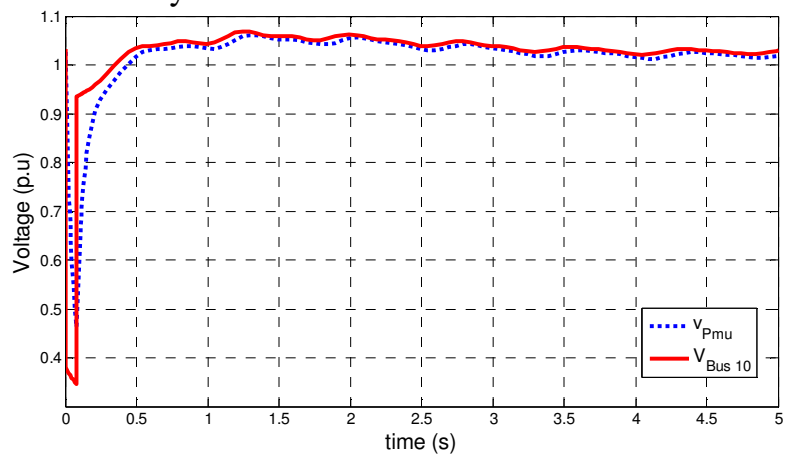

Fig. 6. Actual and PMU measurement for voltage response at bus10 when three phase to ground fault has been applied at bus number five.

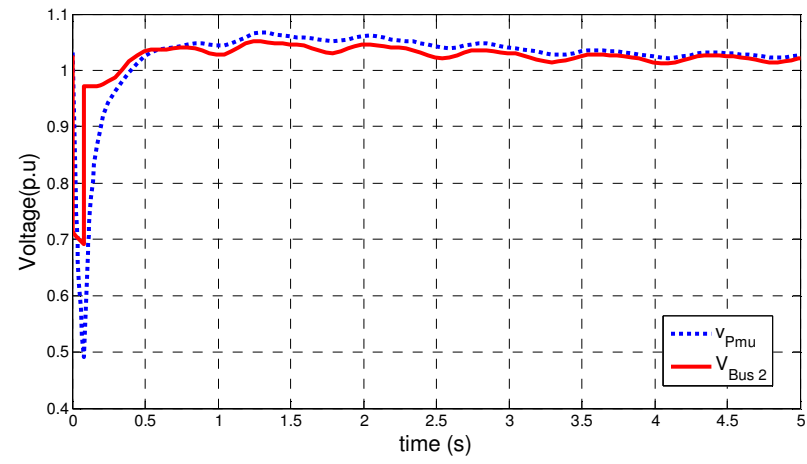

Fig. 7. Actual and PMU measurement for voltage response at bus 2 when a three phase to ground fault has been applied at bus number five

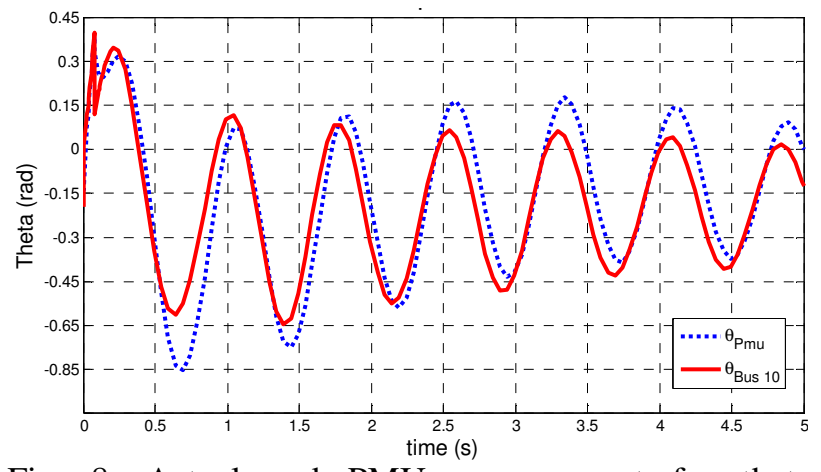

Fig. 8. Actual and PMU measurement for theta response at bus 10 when three phase to ground fault has been applied at bus number five

The difference between PMU measurements of theta response and the actual response of theta at bus 10 after the three phase fault is illustrated in figure 9 , and it is clearly shown that the maximum difference was about $0.08 \mathrm{rad}$ to $+0.06 \mathrm{rad}$. After 10 seconds the difference decreased to about $0.01 \mathrm{rad}$. After 20 seconds there will be no difference at all, it means that at the moment of the fault the error of PMU measurement is relatively high, But it decreases very quickly.

Depending on the previous discussion about the state estimation performance results with PMUs for IEEE 24 bus system, the solution set number ten can be adopted as the optimal location of PMU for the enhancement of the state estimation performance. Therefore, so the proposed method proved its success to be one of the best methods to improve state estimation performance

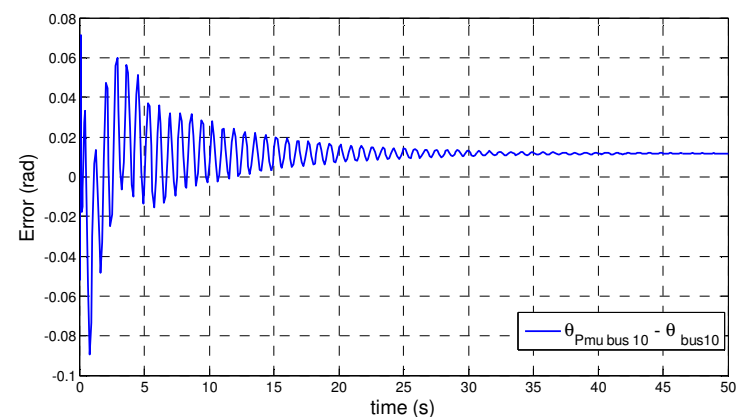

Fig. 9. the difference between theta by PMU measurement and the actual value of theta at bus 10 after the three phase fault at bus 5 .

\section{Conclusions}

This paper suggests a new method to improve the state estimation performance by optimal placement of phasor measurement units (OPP). The proposed PMUs placement algorithm has been tested using IEEE 24 bus system. All simulation has been done by power system analysis toolbox which is operating with MATLAB program. The simulation results show that adding PMUs to the electric power system can significantly improve the state estimation accuracy. The objective of the optimization is the best placement of PMU that improves the state estimation performance. The Root-mean-square deviation RMSD has been used to measure estimation process performance.

The simulation result showed that the proposed method proved its success to be one of the best methods to improve state estimation performance.

The major role for state estimation in the modern power system control and operation is to facilitate the accurate network supervision and monitoring. Thus, an estimation improvement potentially contributes to a more reliable decision making in power system operation, which means, in this case, that the power grid can be operated in a more secured manner.

\section{References}

[1] M. Hurtgen, J. C. Maun, Advantages of power system state estimation using Phasor Measurement Units, in Proceedings of the $16^{\text {th }}$ Power Systems Computation Conference (PSCC), Glasgow, Scotland, UK, 14-18 July 2008; Curran Associates: Red Hook, NY, USA, 2010; pp. 1161-1167. 
[2] A. G. Phadke, Synchronized phasor measurements in power systems, IEEE Comput. Appl. Power, vol. 6, no. 2, pp. 10-15, Apr. 1993.

[3] N. M. Manousakis, G. N. Korres, P. S. Georgilakis, Optimal placement of phasor measurement units: A literature review, in Proceedings of the $16^{\text {th }}$ International Conference on Intelligent System Applications to Power Systems (ISAP), Hersonissos, Greece, 25-28 September 2011; IEEE: Piscataway, NJ, USA, 2011; pp. 1-6.

[4] K. K. More,; H. T. Jadhav, A literature review on optimal placement of phasor measurement units, in Proceedings of the International Conference on Power, Energy and Control (ICPEC), Dindigul, India, 6-8 February 2013; IEEE: Piscataway, NJ, USA, 2013; pp. 220-224.

[5] M. Nazari-Heris, B. Mohammadi-Ivatloo, Application of heuristic algorithms to optimal PMU placement in electric power systems: An updated review, Renew. Sustain. Energy Rev. 2015, pp. 214-228.

[6] K. Negash, B. Khan,; E. Yohannes, Artificial Intelligence Versus Conventional Mathematical Techniques: A Review for Optimal Placement of Phasor Measurement Units, Technol. Econ. Smart Grids Sustain. Energy 2016, 1, 10.

[7] F. Aminifar, M. Fotuhi-Firuzabad, A.Safdarian, Davoudi, A.; M. Shahidehpour, Synchrophasor Measurement Technology in Power Systems: Panorama and State-of-the-Art, IEEE Access 2014, 2, pp. 1607-1628.

[8] A. G. Phadke, J. S. Thorp, Synchronized Phasor Measurements and Their Applications, (1stedn), 2010.

[9] A. Abur, A. G. Exposito, Power System State Estimation: Theory and Implementation, New York. 2004.

[10] K. Zhu, L. Nordstrom, L. Ekstam, Application and analysis of optimum PMU placement methods with application to state estimation accuracy, IEEE Power Energy Society General Meeting, 2009. pp. 1-7.

[11] J. Chen and A. Abur, Placement of PMUs to enable bad data detection in state estimation, IEEE Trans. Power Syst., vol. 21, no. 4, , Nov. 2006, pp. 1608-1615

[12] J. Chen, Y. Liao, Optimal Placement of Phasor Measurement Units for Improving Power System State Estimation Accuracy: A Heuristic Approach, J. Electr. Eng. Electron. Technol. 2012, 1.

[13] M. Shahriar, I. Habiballah, H. Hussein, Optimization of Phasor Measurement Unit (PMU) Placement in Supervisory Control and Data Acquisition (SCADA)-Based Power System for Better State-Estimation Performance, Energies 2018, 11, 570, 10.3390/en11030570. 